\title{
Deconvolution of the relaxations associated with local and segmental motions in poly(methacrylate)s containing dichlorinated benzyl moieties in the ester residue
}

\author{
Gustavo Dominguez-Espinosa and Ricardo Díaz-Calleja \\ Departamento de Termodinámica Aplicada, Escuela Técnica Superior de Ingenieros Industriales (ETSII), \\ Universidad Politécnica de Valencia, Valencia, Spain \\ Evaristo Riande ${ }^{\text {a) }}$ \\ Instituto de Ciencia y Tecnología de Polímeros, Consejo Superior de Investigaciones Científicas (CSIC), \\ 28006 Madrid, Spain \\ Ligia Gargallo and Deodato Radic \\ Departamento de Química Física, Pontificia Universidad Católica de Chile, Santiago, Chile
}

(Received 21 April 2005; accepted 11 July 2005; published online 19 September 2005)

\begin{abstract}
The relaxation behavior of poly(2,3-dichlorobenzyl methacrylate) is studied by broadband dielectric spectroscopy in the frequency range of $10^{-1}-10^{9} \mathrm{~Hz}$ and temperature interval of 303-423 K. The isotherms representing the dielectric loss of the glassy polymer in the frequency domain present a single absorption, called $\beta$ process. At temperatures close to $T_{g}$, the dynamical $\alpha$ relaxation already overlaps with the $\beta$ process, the degree of overlapping increasing with temperature. The deconvolution of the $\alpha$ and $\beta$ relaxations is facilitated using the retardation spectra calculated from the isotherms utilizing linear programming regularization parameter techniques. The temperature dependence of the $\beta$ relaxation presents a crossover associated with a change in activation energy of the local processes. The distance between the $\alpha$ and $\beta$ peaks, expressed as $\log \left(f_{\max ; \beta} / f_{\max ; \alpha}\right)$ where $f_{\max }$ is the frequency at the peak maximum, follows Arrhenius behavior in the temperature range of 310-384 K. Above $384 \mathrm{~K}$, the distance between the peaks remains nearly constant and, as a result, the $\alpha$ onset temperature exhibited for many polymers is not reached in this system. The fraction of relaxation carried out through the $\alpha$ process, without $\beta$ assistance, is larger than $60 \%$ in the temperature range of 310-384 K where the so-called Williams ansatz holds. () 2005 American Institute of Physics. [DOI: 10.1063/1.2013252]
\end{abstract}

\section{INTRODUCTION}

The response of amorphous polymers and, in general, supercooled liquids to a perturbation field appears as a main relaxation called $\alpha$ process or dynamic glass transition, a universal feature of all glass formers. ${ }^{1}$ In the frequency domain, the $\alpha$ or main relaxation is accompanied in most systems by another dynamical process called Johari-Goldstein $\beta$ process located at higher frequencies. ${ }^{2,3}$ The $\alpha$ relaxation is assumed to be produced by the cooperative motions of many particles whereas the secondary relaxation is thought to be more of local origin. In complex systems, such as polymers, other relaxations besides the $\beta$ process may appear at even higher frequencies named $\gamma, \delta$, and so on. ${ }^{4}$

The Adam-Gibbs theory of cooperatively rearranging regions, ${ }^{5}$ the Cohen-Grest free-volume theory for percolation of solid clusters in a liquid matrix, ${ }^{6}$ the Ngai's coupling model, ${ }^{7}$ the Götze's mode-coupling theory, ${ }^{8}$ the Kivelsons' frustration-limited domain (FLD) model, ${ }^{9}$ the Chamberlin's mesoscopic mean-field theory, ${ }^{10}$ the Garrahan-Chandler coarse-grained microscopic model, ${ }^{11}$ and other models ${ }^{12}$ are some of the theoretical approaches to the description of the dynamic glass rubber relaxation. Recently, ${ }^{13}$ a dynamic

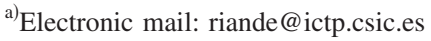

structure of coupled dynamic molecular strings was formulated for supercooled liquids formed by polar small molecules that accounts for the $\alpha$ relaxation. From the Hamiltonian of the rotational degrees of freedom of the system, the strongly correlated supercooled polar liquid state is renormalized to a normal superdipole (SD) liquid state. This scenario describes the following main features of the primary or $\alpha$-relaxation dynamics such as (1) the average relaxation time evolves from a high-temperature Arrhenius to a lowtemperature non-Arrhenius or super-Arrhenius behavior; (2) the relaxation function crosses over from the hightemperature exponential to low-temperature nonexponential form; and (3) the temperature dependence of the relaxation strength shows non-Curie features. According to the model, the crossover between the two first characteristics arises from the transition between the superdipole gas and the superdipole liquid.

Whereas the shape of the $\alpha$ relaxation is essentially independent of temperature, the $\beta$ absorption narrows systematically with increasing temperature. ${ }^{14}$ Another difference between the $\alpha$ and $\beta$ relaxations is related to the temperature dependence of their respective characteristic relaxation times which is governed by the free volume in the first case whereas it is a thermally activated Arrhenius process in the second. As temperature increases, the $\alpha$ relaxation ap- 


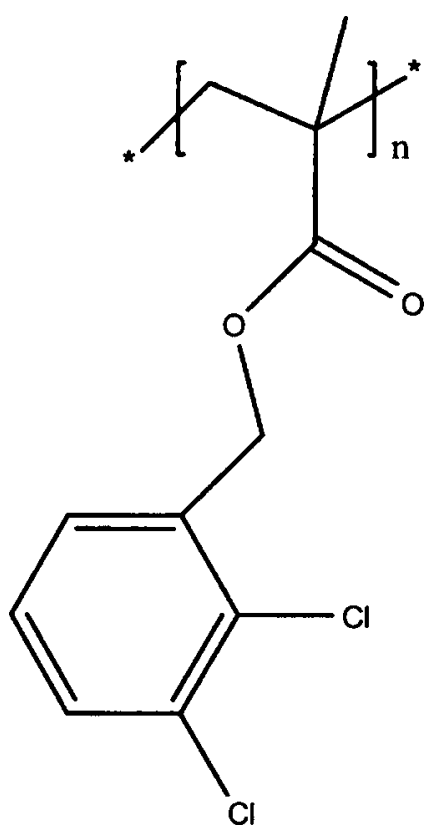

SCHEME 1. Repeating unit of poly(2,3-dichlorobenzyl methacrylate).

proaches the $\beta$ forming a single process called $\alpha \beta$ relaxation. $^{15-17}$ Donth and co-workers ${ }^{18,19}$ investigated in a systematic way the crossover or $\alpha \beta$ splitting region in poly $(n$-alkyl methacrylate)s, an issue of great importance for the understanding of the glass transition. In general, an intensity onset of the cooperative $\alpha$ process, starting from zero at a characteristic onset temperature $T_{\text {on }}$, is observed in all the cases. The rapid increase of the $\alpha$ intensity with decreasing temperature in the vicinity of the $\alpha$ onset was also observed for other polymeric systems. ${ }^{14,18-20}$

This work is a first step to study the influence of the structure on the relaxation behavior of poly(methacryate)s with chlorine-substituted aromatic side groups. The polymer chosen is poly(2,3-dichlorobenzyl methacrylate) (P23DBM), the repeating unit of which is shown in Scheme 1. It may be interesting to compare the relaxation behavior of this polymer with that reported for poly(n-alkyl methacrylate)s to obtain a deeper insight into how slight changes in structure may affect the response of polymeric materials to perturbation fields. The approach taken is based on the determination of the evolution of the retardation spectra with temperature.

\section{EXPERIMENTAL PART}

2,3-dichlorobenzyl methacrylate was obtained by reaction of methacryloyl chloride with 2,3-dichlorobenzyl alcohol in toluene using triethylamine as acid acceptor. Monomers were characterized by ${ }^{1} \mathrm{H}-\mathrm{NMR}$ and ${ }^{13} \mathrm{C}-\mathrm{NMR}$. Polymerization of the monomers in toluene solution was carried out in vacuum, in the vicinity of $328 \mathrm{~K}$, using $\alpha, \alpha^{\prime}$-azobisisobutyronitrile as initiator. Polymers were purified by successive reprecipitations with methanol and further vacuum dried at $303 \mathrm{~K}$ over several days. The glass transition temperature was obtained with a TA Q-10 differential scanning calorimetry (DSC) apparatus at a constant heating rate of $20 \mathrm{~K} / \mathrm{min}$. The $T_{g}$, taken as the temperature at the middle point of the endotherm, was $313 \mathrm{~K}$.

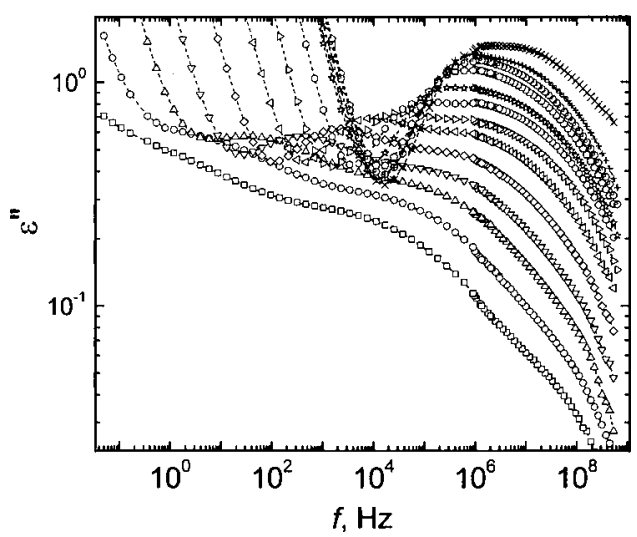

FIG. 1. Isotherms in the frequency domain representing the dielectric loss of poly(2,3-dichlorobenzyl methacrylate), from $303 \mathrm{~K}$ to $423 \mathrm{~K}$ at $10-\mathrm{K}$ steps.

A Novocontrol broadband dielectric spectrometer made up of an Alpha analyzer to carry out measurements from $10^{-1}$ to $10^{6} \mathrm{~Hz}$ and an Agilent $4991 \mathrm{rf}$ analyzer to perform measurements from $10^{6}$ to $3 \times 10^{9} \mathrm{~Hz}$ was used. The dielectric device was coupled to a Quatro temperature controller. Isothermal measurements were carried out from $263 \mathrm{~K}$ to $423 \mathrm{~K}$ in $5-\mathrm{K}$ steps on a molded disk-shaped sample. The diameter of the sample was about $20 \mathrm{~mm}$ for low frequencies and $10 \mathrm{~mm}$ for high frequencies and the thickness in both cases was $0.1 \mathrm{~mm}$. Glass fiber spacers were used to ensure the stability of the sample thickness at high temperatures.

\section{RESULTS}

The loss component of the complex dielectric permittivity in the frequency domain is shown at several temperatures in Fig. 1. The isotherms display a poorly defined $\alpha$ absorption in the low-frequency region associated with the glass transition followed by the $\beta$ relaxation at high frequencies. The conductivity contribution to the $\alpha$ relaxation hides the left side of the dipolar relaxation. The right side of the $\alpha$ absorption is neither well defined as a result of the overlapping of this relaxation with the $\beta$ process. The degree of overlapping between the $\alpha$ and $\beta$ processes increases with increasing temperature until an apparent single $\alpha \beta$ absorption appears.

\section{RETARDATION SPECTRA AND LOSS CURVES DECONVOLUTION}

In general, the $\alpha$ relaxation in the frequency domain is described by the Havriliak-Negami (HN) equation ${ }^{21}$

$$
\varepsilon^{*}(\omega)=\varepsilon_{u}+\frac{\left(\varepsilon_{r}-\varepsilon_{u}\right)}{\left[1+\left(i \omega \tau_{\mathrm{HN}}\right)^{a}\right]^{b}},
$$

where $\varepsilon_{r}$ and $\varepsilon_{u}$ represent, respectively, the relaxed and unrelaxed dielectric permittivities, $\tau_{\mathrm{HN}}$ is a characteristic relaxation time, and the exponents $a$ and $b(0 \leqslant a, a b \leqslant 1)$ are related to the width and asymmetry of the relaxation. It is worth noting that the HN parameters are correlated with those of Kohlrausch ${ }^{22}$ and Williams Watts ${ }^{23}$ (KWW) equation that describes the $\alpha$ relaxation in the time domain. ${ }^{24}$ Secondary relaxations in the frequency domain are well de- 
scribed by the $\mathrm{HN}$ equation with $b=1$. They are symmetric in the logarithmic frequency domain and present a maximum at $\omega_{\max }=1 / \tau_{\mathrm{HN}}$. This symmetric HN function is known as the Cole-Cole equation. The dielectric loss is given by

$$
\frac{\varepsilon^{\prime \prime}(\omega)}{\varepsilon_{r}-\varepsilon_{u}}=\operatorname{Im}\left\{\left[1+\left(i \omega \tau_{\mathrm{HN}}\right)^{a}\right]^{-b}\right\} .
$$

The loss curves described by Eq. (2) display a maximum at a frequency $\omega_{\max }$ given by ${ }^{25}$

$$
\omega_{\max }=\frac{1}{\tau_{\mathrm{HN}}}\left[\sin \left(\frac{\pi a b}{2(b+1)}\right) / \sin \left(\frac{\pi a}{2(b+1)}\right)\right]^{-1 / a} .
$$

Therefore $\omega_{\max } \tau_{\mathrm{HN}}>1$ for the asymmetric $\alpha$ relaxation.

Using the $\mathrm{HN}$ formalism, the dielectric loss in the frequency domain can be written as

$$
\begin{aligned}
\varepsilon^{\prime \prime}(\omega)= & \left(\sigma / \varepsilon_{0} \omega\right)^{s}+\left(\varepsilon_{r}-\varepsilon_{u}\right)_{\alpha} \operatorname{Im}\left\{\left[1+\left(i \omega \tau_{\mathrm{HN}, \alpha}\right)^{a}\right]^{-b} a\right\} \\
& +\left(\varepsilon_{r}-\varepsilon_{u}\right)_{\beta} \operatorname{Im}\left\{\left[1+\left(i \omega \tau_{\mathrm{HN}, \beta}\right)^{\alpha_{\beta}}\right]^{-1}\right\},
\end{aligned}
$$

where $\sigma$ is the ionic conductivity, $\varepsilon_{0}$ is the permittivity in vacuum, and the exponent $s$ measures the departure of the conductive contribution to the loss from pure ionic conductivity $(s=1)$ as a consequence of interfacial blocking electrode effects or other phenomena. The deconvolution of the $\alpha$ and $\beta$ processes in the isotherms of Fig. 1 by means of Eq. (4) presents some difficulties as a consequence of the rather large number of fitting parameters involved, on the one hand, and the high degree of overlapping even at temperatures close to $T_{g}$, on the other hand. Actually, one would expect different combinations leading to the deconvolution of the isotherms in such a way that to discriminate the correct one could present some difficulties.

The ability to resolve two overlapping $\alpha$ and $\beta$ relaxations becomes easier using retardation spectra because the peak associated to each process is narrower in the time domain than in the frequency domain. This extreme can easily be understood if we consider that the Debye relaxation in the time domain is a Dirac delta function whereas the half-width of the relaxation in the frequency domain is slightly greater than one decade. According to the linear phenomenology of dielectrics, the dielectric loss can be written as ${ }^{26}$

$$
\varepsilon^{\prime \prime}(\omega)=\left(\frac{\sigma}{\varepsilon_{0} \omega}\right)^{s}+\int_{-\infty}^{\infty} L(\ln \tau) \frac{\omega \tau}{1+\omega^{2} \tau^{2}} d \ln \tau,
$$

where $L$ is the retardation spectrum. The inversion of Eq. (5) can be carried out by obtaining different $L$ 's for various physically admissible values of $s$ (in this system, $s$ lies in the range of $0.8<s \leqslant 1$ ) and selecting the one that optimizes this equation. This procedure transforms the initial nonlinear problem into a linear one, i.e., into solving a first kind Fredholm integral equation. Let us assume we want to calculate the spectrum for some discrete times $L\left(\ln \tau_{j}\right)$ $(j=1,2, \ldots, m-1)$ and $\sigma$ from a set of $n$ measurements of $\varepsilon^{\prime \prime}\left(\omega_{i}\right)$ at angular frequencies $\omega_{i}(i=1,2, \ldots, n)$ with uncertainties $\rho_{i}$. Equation (5) can be written in an approximate way $\mathrm{as}^{20}$

$$
\frac{\varepsilon^{\prime \prime}\left(\omega_{i}\right)}{\rho_{i}} \cong\left[\left(\frac{\sigma}{\varepsilon_{0} \omega_{i}}\right)^{s}+\sum_{k=1}^{m} R_{i k} L\left(\ln \tau_{k}\right)\right] / \rho_{i},
$$

where

$$
R_{i k}=\frac{\omega_{i} \tau_{k}}{1+\omega_{i} \tau_{k}^{2}} \ln \left(\frac{\tau_{k+1}}{\tau_{k-1}}\right)^{1 / 2} .
$$

It is convenient to write Eq. (6) in matricial form as

$$
\begin{aligned}
{\left[\begin{array}{l}
\varepsilon^{\prime \prime}\left(\omega_{1}\right) / \rho_{1} \\
\varepsilon^{\prime \prime}\left(\omega_{2}\right) / \rho_{2} \\
\ldots \\
\varepsilon^{\prime \prime}\left(\omega_{n}\right) / \rho_{n}
\end{array}\right] } & \cong\left[\begin{array}{cc}
R_{11} / \rho_{1} R_{12} / \rho_{1} \ldots R_{1 m-1} / \rho_{1} & \omega_{1}^{-s} / \rho_{1} \\
R_{21} / \rho_{2} R_{22} / \rho_{2} \ldots R_{2 m-1} / \rho_{2} & \omega_{2}^{-s} / \rho_{2} \\
\ldots & \\
R_{n 1} / \rho_{n} R_{n 2} / \rho_{n} \ldots R_{n m-1} / \rho_{n} & \omega_{n}^{-s} / \rho_{n}
\end{array}\right] \\
& \times\left[\begin{array}{l}
L\left(\tau_{1}\right) \\
L\left(\tau_{2}\right) \\
\ldots \\
L\left(\tau_{m-1}\right) \\
\left.\left(\frac{\sigma}{\varepsilon_{0}}\right)^{s}\right],
\end{array}\right.
\end{aligned}
$$

or in a more compact form:

$\varepsilon^{\prime \prime} \cong \mathbf{R L}$.

The solution of the ill-conditioned Eq. (5) is similar to that of the also ill-posed quadratic programming problem $\min \left|\boldsymbol{\varepsilon}^{\prime \prime}-\mathbf{R L}\right|^{2}$, equivalent to ${ }^{27}$

$$
\min \left|\boldsymbol{\varepsilon}^{\prime \prime}-\mathbf{R} \mathbf{L}\right|^{2}+\lambda\left(\mathbf{L}^{T} \mathbf{H L}\right),
$$

where $\lambda(>0)$ is known as the regularization parameter and $\mathbf{H}$ is a definite positive quadratic form. The main idea underlying this transformation is that when a quadratic minimization principle is combined with a quadratic constraint, and both are positive, only one of the two needs to be no degenerate for the overall problem to be well posed.

The election of the $\mathbf{H}$ matrix must be based on the a priori knowledge of the solution. If the solution is thought to be piecewise linear, a good choice is $\mathbf{H}=\mathbf{B}^{T} \mathbf{B}$ where $\mathbf{B}$ is the $(m-2) \times m$ matrix $^{27}$

$$
\mathbf{B}=\left[\begin{array}{ccccccc}
-1 & 2 & -1 & 0 & 0 & \ldots & 0 \\
0 & -1 & 2 & -1 & 0 & \ldots & 0 \\
\ldots & & & & & & \\
0 & \ldots \ldots & -1 & 2 & -1 & 0 \\
0 & \ldots \ldots & 0 & -1 & 2 & -1
\end{array}\right]
$$

and $\mathbf{B}^{T}$ reads for the transpose of the matrix $\mathbf{B}$. The solutions corresponding to different values of the regularization parameter $\lambda$ were obtained by means of an iterative technique (conjugated gradient) that allows the utilization of the projection onto convex set (POCS) methods for achieving a non-negative solution. The best values of $\lambda$ were determined by means of the Morozov's discrepancy method. ${ }^{28}$ The spectrum was calculated from values of $\tau$ lying two decades above and below the experimental range. Because the intensity of Debye relaxations falls to the $1 \%$ of its maximum value for $\omega \tau=10^{ \pm 2}$, the contributions to the experimental results of the hypothetical relaxations that are two decades 


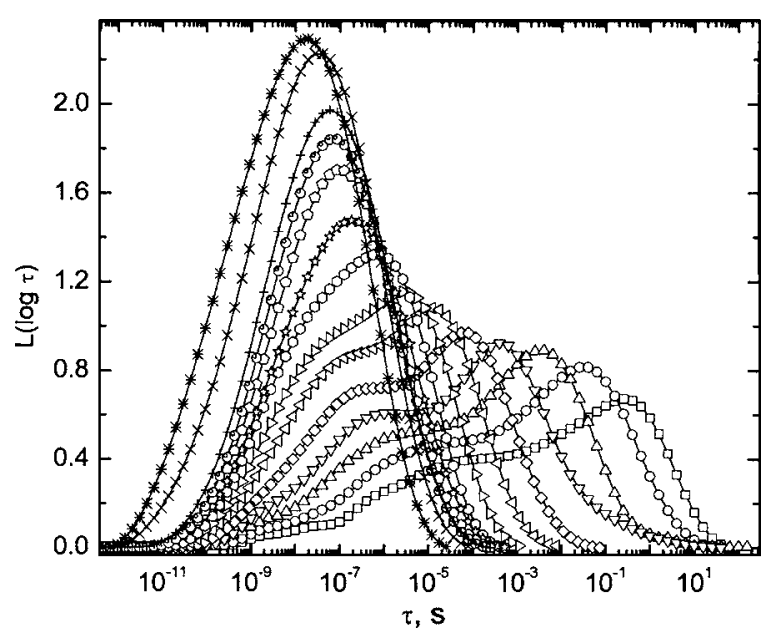

FIG. 2. Distribution of retardation times from $303 \mathrm{~K}$ to $423 \mathrm{~K}$, at $10-\mathrm{K}$ steps, for poly(2,3-dichlorobenzyl methacrylate).

apart from the interval of interest can be neglected. It is important to remark that this modus operandi that allows minimizing aliasing effects does not require any assumption about the values of $\varepsilon^{\prime \prime}$ out of the experimental window.

The retardation spectrum formalism has previously been used to analyze dielectric spectroscopy results, for instance, with the CONTIN program ${ }^{29}$ (see, for example, Ref. 42 in Ref. 26). This procedure has some drawbacks when compared with that used in this paper that are related to the way of dealing with the conductivity contribution, to the determination of a nonaliased solution, to the method utilized to determine the regularization parameter and to the computational efficiency of the general methods for solving quadratic programming problems.

The retardation spectra from 303 to $423 \mathrm{~K}$, calculated in $10-\mathrm{K}$ steps by the method described above, are shown in Fig. 2. By subtracting the conductivity contribution to the dielectric loss, the dipolar relaxation is obtained. The pertinent isotherms are shown in Fig. 3 where the overlapping between the $\alpha$ and $\beta$ relaxations becomes clearer than in Fig. 1.

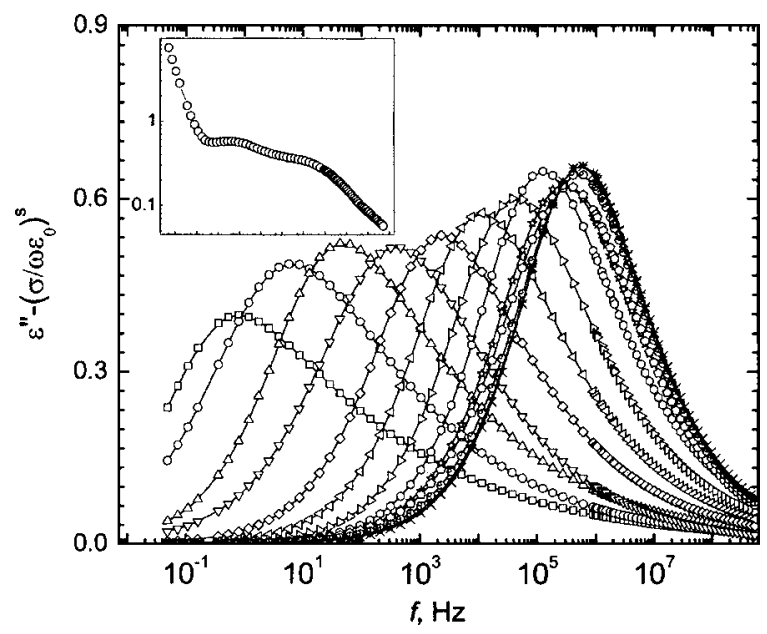

FIG. 3. The same as Fig. 1 without the ionic conductivity contribution to the loss. The inset shows the isotherm at $323 \mathrm{~K}$ with the ionic conductivity contribution to the loss.

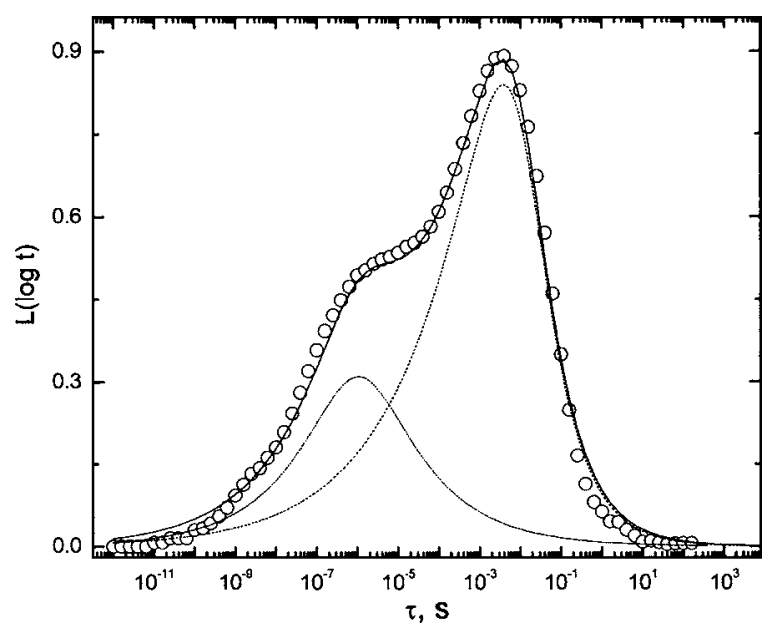

FIG. 4. Deconvolution of the calculated retardation spectrum (circles) at $323 \mathrm{~K}$ for poly(2,3-dichlorobenzyl methacrylate). The continuous line is the sum of the contributions of the $\alpha$ (dash line) and $\beta$ (dot line) relaxations to the spectrum.

Deconvolutions of the retardation spectra were performed by using for the inverse of the $\mathrm{HN}$ empirical equation the following expression: ${ }^{30}$

$$
L(\ln \tau)=\frac{1}{\pi} \frac{\left(\tau / \tau_{0}\right)^{a b} \sin b \theta}{\left[\left(\tau / \tau_{0}\right)^{2 a}+2\left(\tau / \tau_{0}\right)^{a} \cos a \pi+1\right]^{b / 2}} .
$$

The parameter $\theta$ in Eq. (12) can be written as

$$
\theta=\arctan \left(\frac{\sin \pi a}{\left(\tau / \tau_{a}\right)^{a}+\cos \pi a}\right),
$$

if the argument of arctan is positive. If it is negative, then

$$
\theta=\arctan \left(\frac{\sin \pi a}{\left(\tau / \tau_{0}\right)^{a}+\cos \pi a}+\pi\right) .
$$

The $\beta$ absorption was analyzed in terms of the FuossKirkwood equation given by ${ }^{31}$

$$
\varepsilon^{\prime \prime}(\omega)=\frac{2 \varepsilon_{\max }^{\prime \prime}\left(\omega / \omega_{\max }\right)^{m}}{1+\left(\omega / \omega_{\max }\right)^{2 m}},
$$

where $m$ is an empirical parameter whose value lies in the interval $0<m \leqslant 1$ and $\omega_{\max }$ is the frequency at the peak maximum at which the loss is $\varepsilon_{\max }^{\prime \prime}$. The lower $m$ is the wider the relaxation is and for a Debye-type process $m=1$. The analytical expression for the retardation spectrum of a relaxation obeying FK behavior is ${ }^{32}$

$$
L(\ln \tau)=\frac{1}{\pi} \frac{m \cosh \left[m \ln \left(\tau / \tau_{F K}\right) \cos (\pi m / 2)\right]}{\left.\sinh ^{2}\left[m \ln \left(\tau / \tau_{F K}\right)\right]+\cos ^{2}(\pi m / 2)\right]} .
$$

For illustrative purposes, the retardation spectrum of the dielectric loss at $323 \mathrm{~K}$ as well as the spectra of the $\alpha$ and $\beta$ processes obtained by means of Eqs. (12) and (16), respectively, are shown in detail in Fig. 4. It can be seen that the sum of the contributions of the $\alpha$ and $\beta$ relaxations coincides pretty well with the overall retardation spectrum. As expected, the $\alpha$ peak is asymmetric, dropping more slowly with decreasing time on the left-hand side than on the right-hand side. Figure 5 shows the loss isotherm at 323 recalculated from the spectrum together with the deconvoluted $\beta$ and $\alpha$ 


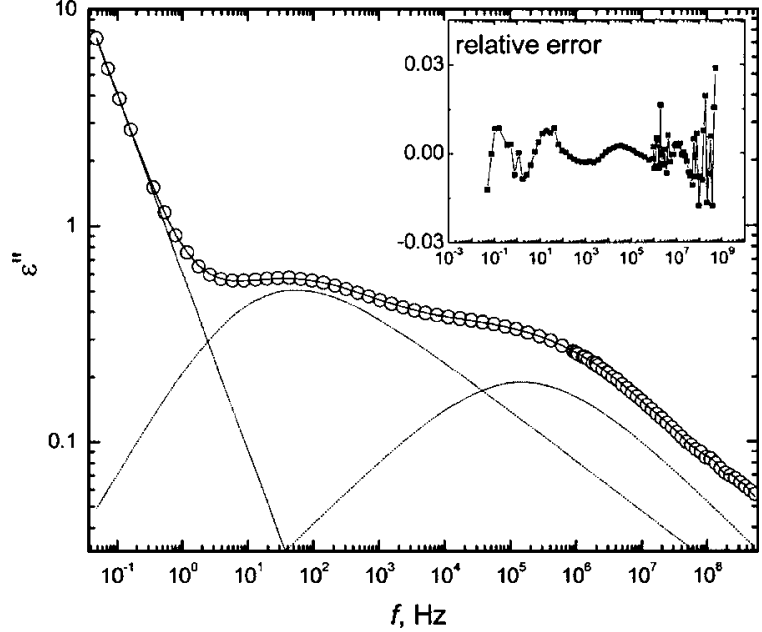

FIG. 5. Isotherm representing the dielectric loss in the frequency domain at $323 \mathrm{~K}$ for poly(2,3-dichlorobenzyl methacrylate). The circles and the continuous line represent, respectively, the experimental data and the sum of the $\alpha, \beta$, and conductivity contributions to the loss. In the inset, the relative error $\left(\varepsilon_{\mathrm{EXP}}^{\prime \prime}-\varepsilon_{\mathrm{CALC}}^{\prime \prime}\right) / \varepsilon_{\mathrm{EXP}}^{\prime \prime}$ is shown.

relaxations. The fitting is rather good as the errors plotted in the inset of Fig. 5 demonstrate. Only at extreme high frequencies and at frequencies where the data proceeding from different instruments intersect, the errors seem to be somewhat larger. The deconvoluted $\alpha$ and $\beta$ retardation spectra covering the range of $303-343 \mathrm{~K}$ in steps of $10 \mathrm{~K}$ are shown in Figs. 6 and 7, respectively. As Fig. 7 suggests, the width of the $\beta$ peaks decreases with increasing temperature though the decrease is accompanied of an augment of intensity. The deconvoluted $\alpha$ relaxation does not seem to be so affected by temperature as the $\beta$ process is.

\section{RELAXATION STRENGTHS}

Besides the mean relaxation time, the strength and the shape characterize the relaxations. The relaxation strength can be calculated from the retardation spectra by means of the classical expression

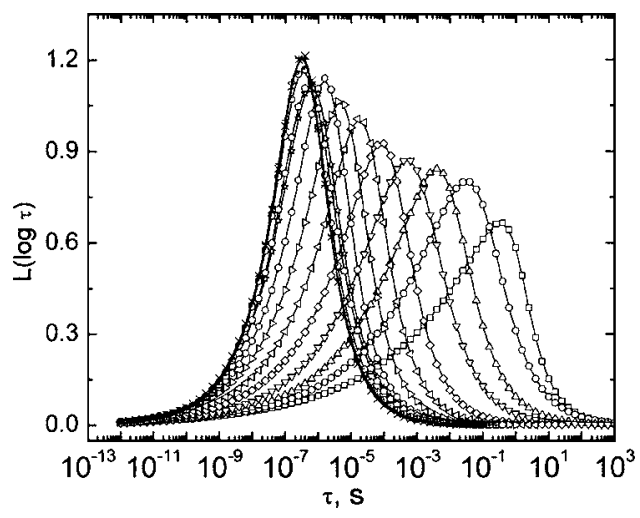

FIG. 6. Retardation spectra for the $\alpha$ relaxation from $313 \mathrm{~K}$ to $423 \mathrm{~K}$, at 10 -K steps, for poly(2,3-dichlorobenzyl methacrylate).

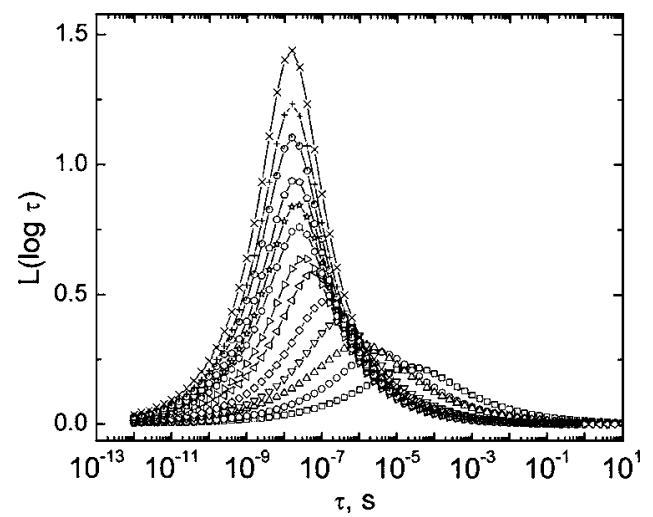

FIG. 7. Retardation spectra for the $\beta$ relaxation from $303 \mathrm{~K}$ to $423 \mathrm{~K}$, at 10-K steps, for poly(2,3-dichlorobenzyl methacrylate).

$$
\varepsilon_{r ; i}-\varepsilon_{u ; i}=\int_{-\infty}^{\infty} L_{i}(\ln \tau) d \ln \tau
$$

where the subscript $i$ refers to the type of relaxation $(\alpha$ or $\beta)$. The pertinent results, shown in Fig. 8, indicate that the strength of the $\alpha$ relaxation decreases with increasing temperature while that of the $\beta$ process experiences a significant increase as temperature augments, this increase being even larger in the region where the $\alpha \beta$ process occurs. As a result, the total dielectric strength increases rather steeply at high temperatures.

The values of $m$ fitting Eq. (16) to the $\beta$ retardation spectra, collected in Fig. 9, slightly increase with temperature, specifically from 0.23 at $303 \mathrm{~K}$ up to 0.38 at $423 \mathrm{~K}$. The values of the $a$ and $b$ exponents fitting the HN equation to the $\alpha$ relaxation are also plotted as a function of temperature in Fig. 9. The exponent $a$ remains nearly constant in the whole temperature range while the exponent $b$ which accounts for the skewness of the Cole-Cole complex plot (it decreases with increasing skewness) augments with increasing temperature reaching a ceiling value of $0.7-0.8$ at high temperature.

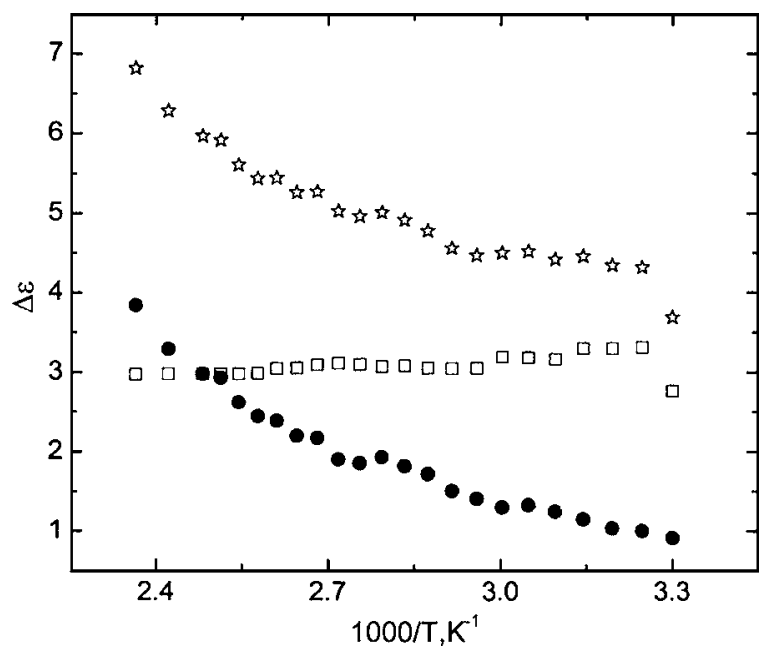

FIG. 8. Relaxation strength of the $\alpha$ relaxation ( $\square), \beta$ absorption $(\bullet)$, and $\alpha+\beta$ process (放) for poly(2,3-dichlorobenzyl methacrylate). 


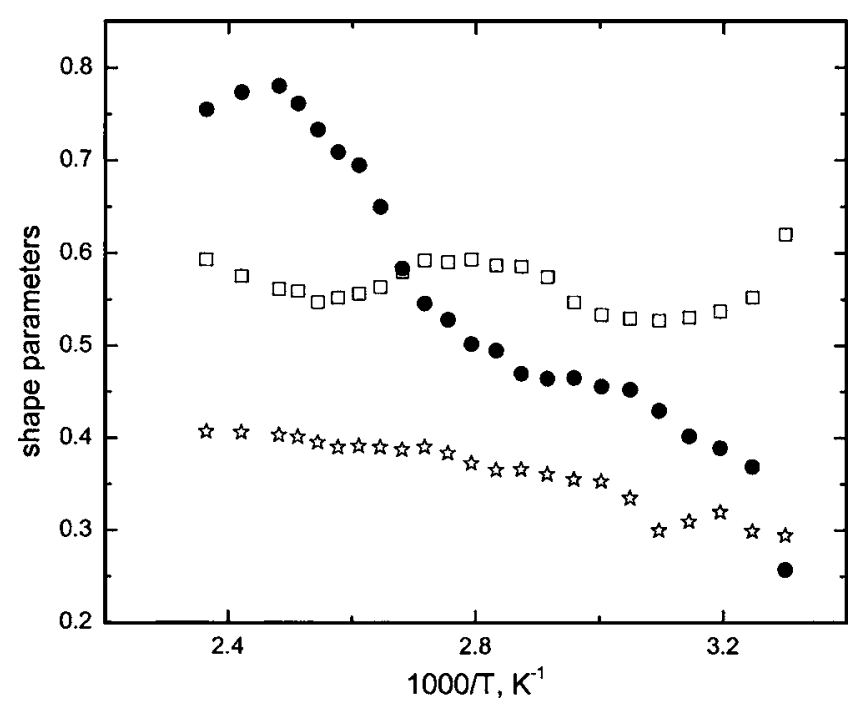

FIG. 9. Temperature dependence of the fitting parameters of the $\alpha$ and $\beta$ relaxations for poly(2,3-dichlorobenzyl methacrylate). The symbols (i $)$, $(\square)$, and $(\bullet)$ refer, respectively, to the $m$ parameter of the Fuoss-Kirkwood equation and to the $a$ and $b$ exponents of the Havriliak-Negami equation.

\section{TEMPERATURE DEPENDENCE OF THE RELAXATIONS}

Following the Arrhenius formalism, the natural logarithm of the mean retardation times associated with the maxima of the deconvoluted $\alpha$ and $\beta$ peaks of the retardation spectra are plotted against the reciprocal of the absolute temperature in Fig. 10. Coming from the low-temperature side, the Arrhenius plot for the $\beta$ peak exhibits a crossover just at the temperature at which the $\alpha \beta$ relaxation is apparently formed. Below the crossover temperature $T_{\mathrm{co}}$ the activation energy of the $\beta$ process is $122 \pm 3 \mathrm{~kJ} / \mathrm{mol}$ whereas the value of this parameter is only $57 \pm 2 \mathrm{~kJ} / \mathrm{mol}$ above $T_{\mathrm{co}}$. During cooling, the $\alpha$ relaxation deviates significantly from a thermally activated process, and the temperature dependence of the retardation times is described by the empirical VogelFulcher-Tammann-Hesse (VFTH) equation ${ }^{33}$

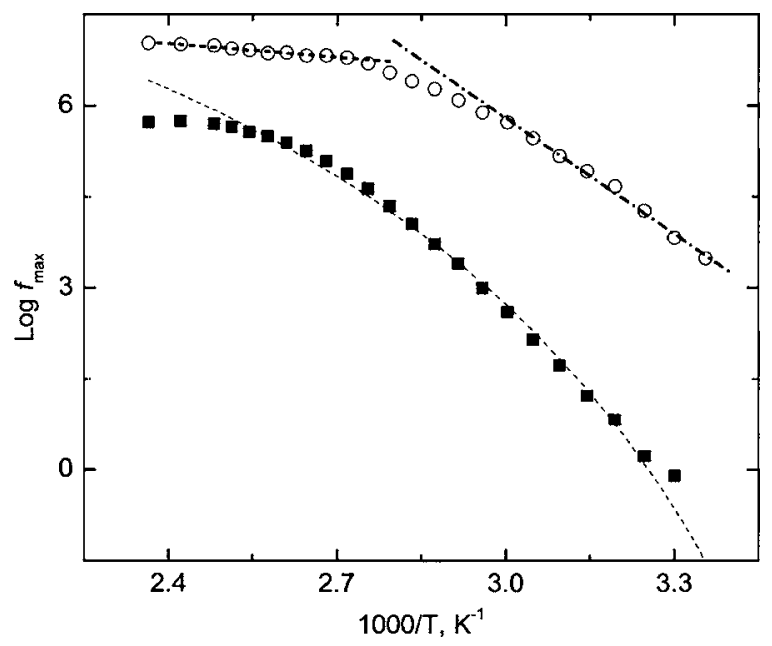

FIG. 10. Arrhenius plot for the $\alpha$ and $\beta$ relaxations of poly(2,3dichlorobenzyl methacrylate).

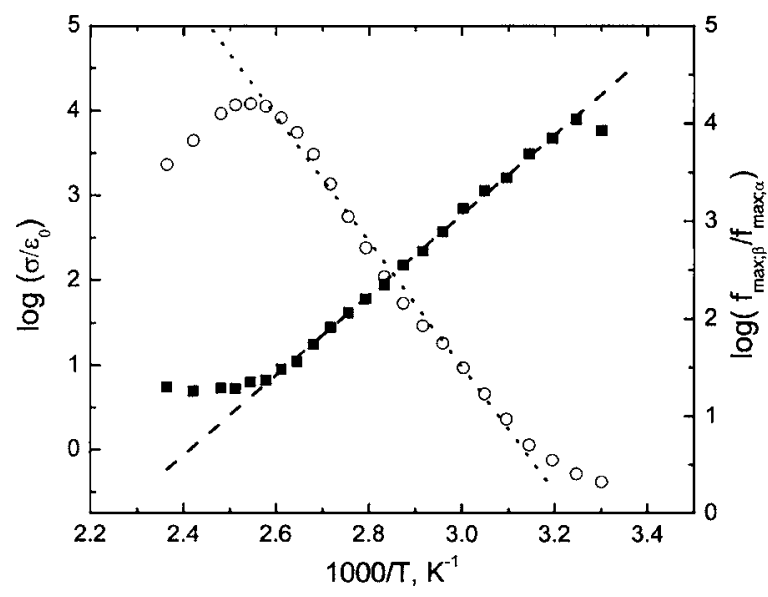

FIG. 11. Arrhenius plot showing $(\bigcirc)$ the temperature dependence of the ionic conductivity in $\Omega^{-1} \mathrm{~m}^{-1}$ and $(\checkmark)$ the distance between the maxima of the $\beta$ and $\alpha$ peaks, expressed as $\log \left(f_{\max ; \beta} / f_{\max ; \alpha}\right)$, for $\operatorname{poly}(2,3-$ dichlorobenzyl methacrylate).

$$
\tau=\tau_{0} \exp \left(\frac{m^{\prime}}{T-T_{V}}\right),
$$

where $\tau_{0}$ is a prefactor of the order of picoseconds and $T_{V}$ is the Vogel temperature where the extrapolated relaxation time diverges. ${ }^{1,34}$ The fact that Eq. (18) also fits the temperature dependence of the viscosity suggests that the structural or $\alpha$ relaxation is a precursor of both the glassy state and the viscous flow. Equation (18) fits the experimental results for values of $m^{\prime}$ and $T_{V}$ equal to $1200 \pm 190$ and $250 \pm 10 \mathrm{~K}$, respectively. Angell ${ }^{34,35}$ has introduced the fragile concept in glasses by defining $m^{\prime}=D T_{V}$ where $D$ is the so-called fragile parameter that expresses the departure of supercooled liquids from Arrhenius behavior. Fragile glass formers ${ }^{1}$ are characterized for having a fragile parameter $D$ lower than 10 , while the so-called strong supercooled liquids have values of $D$ higher than 10 . The fact that $D \cong 5$ for poly(2,3 dichlorobenzyl methacrylate) reveals a rather fragile character for this glass former.

The mean retardation time can also be written in terms of the empirical free-volume Doolittle equation ${ }^{36}$

$$
\tau=\tau_{0} \exp \left(\frac{B}{\Phi}\right),
$$

where $\Phi$ is the free-volume fraction and $B$ is a parameter close to unit related to $\nu^{*} / \nu_{m}, \nu^{*}$ and $\nu_{m}$ being, respectively, the critical volume necessary for a relaxation process to take place and the volume of the segments intervening in the relaxation. ${ }^{37}$ By comparing Eqs. (18) and (19) one finds

$$
\frac{\Phi_{g}}{B}=\frac{m}{T_{g}-T_{V}},
$$

where $\Phi_{g}$ is the free-volume fraction at $T_{g}$. Accordingly, the $\Phi_{g} / B$ ratio is about 0.048 , nearly two times larger than the value found for this quantity in most polymers which lies in the range of $0.025 \pm 0.005$. If the iso-free-volume theory holds $^{38}$ the fact that $\Phi_{g} / B>0.025$ means that $\nu^{*}<\nu_{m}$.

The temperature dependence of the ionic conductivity expressed in terms of $\sigma / \varepsilon_{0}$ is shown in Fig. 11. It can be seen that the conductivity obeys Arrhenius behavior with an acti- 


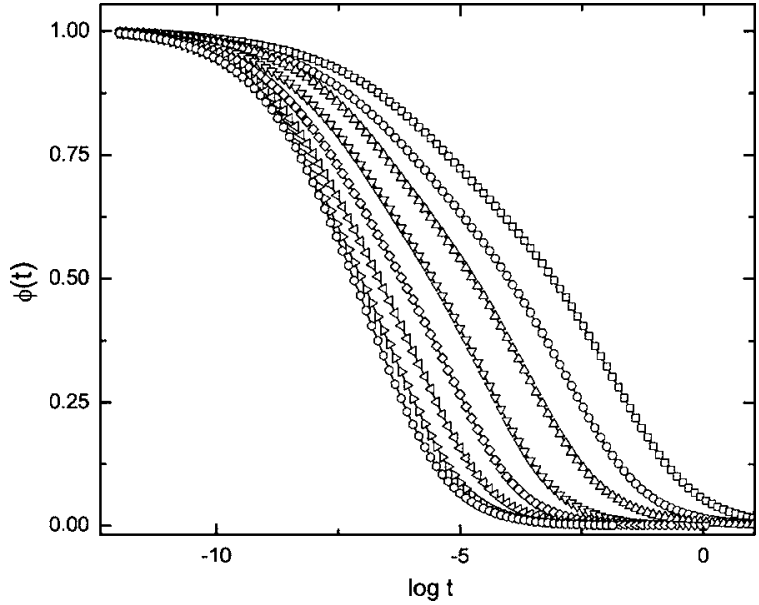

FIG. 12. Total decay function for the relaxation of poly(2,3-dichlorobenzyl methacrylate) from $313 \mathrm{~K}$ to $383 \mathrm{~K}$, at $10-\mathrm{K}$ steps, for poly $(2,3-$ dichlorobenzyl methacrylate).

vation energy of the order of $140 \pm 2 \mathrm{~kJ} \mathrm{~mol}^{-1}$. Therefore ionic transport is carried out overcoming energy barriers of the same order as those of the low-temperature $\beta$ process.

\section{WILLIAMS CORRELATION FUNCTION}

The normalized time dipole correlation function can be calculated from the retardation spectra by means of the following expression:

$$
\phi(t)=\frac{\int_{-\infty}^{\infty} L(\ln \tau) e^{-t / \tau} d \ln \tau}{\int_{-\infty}^{\infty} L(\ln \tau) d \ln \tau} .
$$

The pertinent decay functions, shown at the temperatures of interest in Fig. 12, drop faster the higher the temperature is. Williams ${ }^{15}$ views the $\beta$ relaxation as due to restricted motions in a range of local environments, which permit a partial relaxation. This limited relaxation leads to an initial fall in the time dipole correlation function $\phi(t)$. At longer times, micro-Brownian motions cause fluctuations of local environments that lead to the total randomization of the dipole vector and as result $\phi(t)$ becomes zero. By neglecting cross correlation contributions, Williams's assumption can be expressed as ${ }^{15,17}$

$$
\phi(t)=\phi_{\alpha}(t)\left[f_{\alpha}+\left(1-f_{\alpha}\right) \phi_{\beta}(t)\right],
$$

where $\phi_{\alpha}(t)$ and $\phi_{\beta}(t)$ are, respectively, the normalized correlation functions for the $\alpha$ and $\beta$ relaxations and $1-f_{\alpha}$ represents the fraction of mechanisms that relax via the combined $\alpha$ and $\beta$ processes. This approach is known as Williams-Watts (WW) ansatz. The $\alpha$ and $\beta$ relaxations are coupled phenomena because all of them presumably involve motions of the dipoles of the side group. When the $\alpha$ and $\beta$ processes are well separated, the relaxation of the $\alpha$ process is not important until the total relaxation of the secondary absorption is not complete; i.e., $\phi_{\alpha}(t) \approx 1$ when $\phi_{\beta}(t) \approx 0$. In this situation, Eq. (22) becomes

$$
\phi(t)=f_{\alpha} \phi_{\alpha}(t)+\left(1-f_{\alpha}\right) \phi_{\beta}(t) .
$$

In the frequency domain, Eq. (22) can be written as ${ }^{39}$

$$
\begin{aligned}
\phi^{*}(\omega)=\frac{\varepsilon^{*}(\omega)-\varepsilon_{u}}{\varepsilon_{r}-\varepsilon_{u}} & =L_{i \omega}\left(-\frac{d \phi(t)}{d t}\right) \\
& =f_{\alpha} \phi_{\alpha}^{*}(\omega)+\left(1-f_{\alpha}\right) \phi_{\alpha}^{*} \phi_{\beta}^{*},
\end{aligned}
$$

where $L_{i \omega}$ is the Laplace operator and $\phi_{k}^{*}(\omega)=L_{i \omega}\left(-d \phi_{k} / d t\right)$, the subscript $k$ meaning $\alpha$ or $\beta$. The normalized $\phi_{k}(t)$ function is obtained by substituting $L(\ln \tau)$ in Eq. (21) by $L_{k}(\ln \tau)$. The product $\phi_{\alpha}^{*}(\omega) \phi_{\beta}^{*}(\omega)$ can be expressed in terms of the retardation spectrum by ${ }^{39}$

$$
\begin{aligned}
\phi_{\alpha}^{*}(\omega) \phi_{\beta}^{*}(\omega)= & \frac{\int_{-\infty}^{\infty} L_{\alpha}(\ln \tau)}{\int_{-\infty}^{\infty} L_{\alpha}(\ln \tau) d \ln \tau}\left[\frac{1}{\int_{-\infty}^{\infty} L_{\beta}\left(\ln \tau^{\prime}\right) d \ln \tau^{\prime}}\right. \\
& \times \int_{-\infty}^{\infty} L_{\beta}\left(\ln \tau^{\prime}\right) \frac{1}{1+i \omega\left(\frac{1}{\tau}+\frac{1}{\tau^{\prime}}\right)^{-1}} d \ln \tau^{\prime} \\
& \times d \ln \tau .
\end{aligned}
$$

In strongly overlapped systems, such as that occurs in the merging temperature region, the fraction of relaxation taking place exclusively through the $\alpha$ process should be negligible. ${ }^{14}$ In this situation, the dielectric loss for strongly overlapped relaxations should approximately be given by

$$
\begin{aligned}
\varepsilon^{\prime \prime}(\omega) \cong & \int_{-\infty}^{\infty} L_{\alpha}(\ln \tau) \\
& \times\left[\int_{-\infty}^{\infty} L_{\beta}\left(\ln \tau^{\prime}\right) \frac{\omega\left(\frac{1}{\tau}+\frac{1}{\tau^{\prime}}\right)^{-1}}{1+\omega^{2}\left(\frac{1}{\tau}+\frac{1}{\tau^{\prime}}\right)^{-2}} d \ln \tau^{\prime}\right] d \ln \tau .
\end{aligned}
$$

Since a strong correlation between the main and secondary relaxations in nearly the whole range of temperatures above $T_{g}$ exists, we proceeded to the analysis of the dielectric loss using Eq. (26). We found that the loss isotherms lying in the range of 313-383 $\mathrm{K}$ are not fitted by this equation in spite of the fact that, at first sight, a severe overlapping between the $\alpha$ and $\beta$ relaxations seems to occur. However, good fitting is obtained if Eq. (24) instead of Eq. (26) is used, as the plot of Fig. 13 illustrates. The fraction $f_{\alpha}$ in Eq. (24) represents the fraction of polarization relaxing through the $\alpha$ process alone, with no $\beta$ assistance. Similar behavior has been reported by Donth and co-workers for some poly(n-alkyl methacrylate)s. ${ }^{19}$ An Arrhenius plot of the pertinent values of $f_{\alpha}$ fitting the experimental results is shown in Fig. 14. It can be seen that $f_{\alpha}$ is roughly a linear decreasing fraction of the reciprocal of temperature but the decrease is rather small if we compare it with that observed in other polymers. ${ }^{14,20}$ Above $383 \mathrm{~K}$, just at the temperature at which the total strength of the relaxation experiences a sharp increase with increasing temperature, the Williams ansatz no longer holds, as the results of Fig. 15 corresponding to $393 \mathrm{~K}$ demonstrate. Notice that the fitting is rather poor in the central region of the isotherm. 


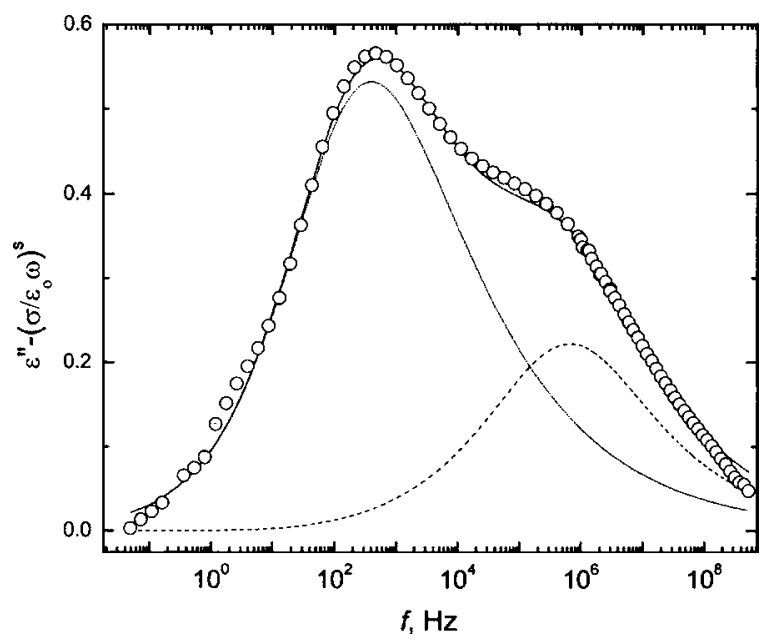

FIG. 13. Values of the dipolar dielectric loss at $333 \mathrm{~K}$ for poly $(2,3$ dichlorobenzyl methacrylate): (circles and continuous line) experimental and calculated results using the Williams-Watts ansatz [Eq. (23)]. The dash curves correspond to the contributions of the $\alpha$ (low frequency) and $\beta$ (high frequency) relaxations.

\section{DISCUSSION}

The dipole associated with the ester group of poly(2,3dichlorobenzyl methacrylate) has a value of $1.78 \mathrm{D}$ and it forms an angle of $123^{\circ}$ with the $\mathrm{C}\left(\mathrm{CH}_{3}\right)-\mathrm{C}(\mathrm{O}) \mathrm{O}$ bond. Two dipoles more, each one of $2.00 \mathrm{D}$, are located in positions 2 and 3 in the phenyl ring. ${ }^{40}$ With the exception of the $\mathrm{C}(\mathrm{O})-\mathrm{O}$ bond of the ester group, which is restricted to the trans state, motions about the rest of bonds of the side groups may give rise to considerable dielectric activity. By similarity with what occurs with poly $\left(n\right.$-alkyl methacrylate) ${ }^{17,41}$ a great part of the $\beta$ relaxation can be postulated to arise from motions about the $\mathrm{C}-\mathrm{C}(\mathrm{O})$ bond connecting the dipole associated with the $\mathrm{C}-\mathrm{C}(\mathrm{O}) \mathrm{OCH}_{2}$ to the main chain, and the rest by motions about the $\mathrm{O}-\mathrm{CH}_{2}-\mathrm{C}^{\text {ar }}$ moiety, where $\mathrm{C}^{\text {ar }}$ represents an aromatic carbon bond.

The Arrhenius plot of Fig. 14 reveals the existence of a crossover temperature that apparently marks the frontier between two different mechanisms for the $\beta$ process. Below

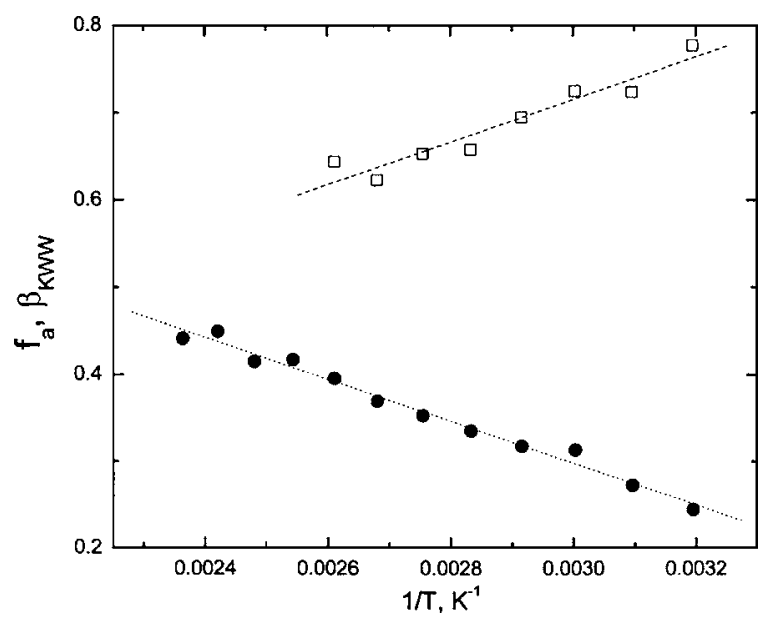

FIG. 14. Arrhenius plots for both the fraction of the $\alpha$ process relaxing alone, without $\beta$ assistance $(\square)$, and the stretch exponent of the KWW equation (-) in the range of temperatures $313-383 \mathrm{~K}$.

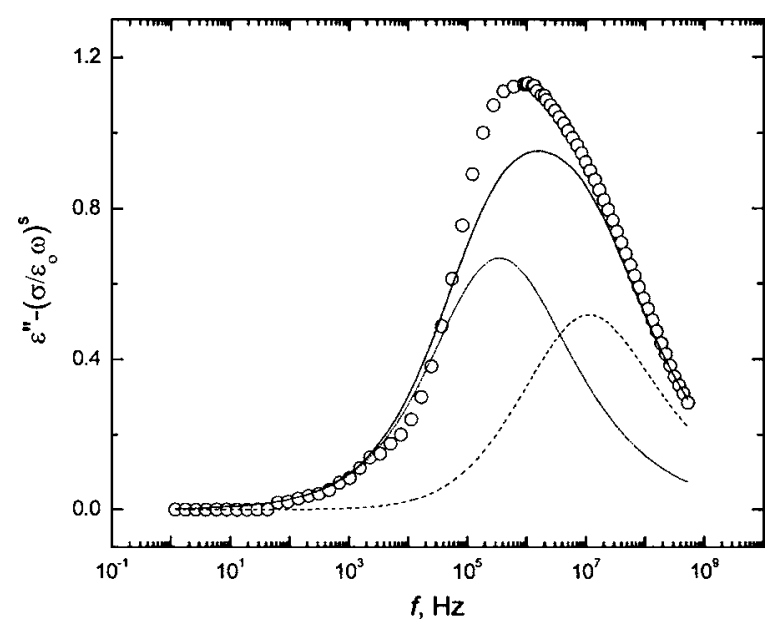

FIG. 15. Values of the dipolar dielectric loss at $393 \mathrm{~K}$ for poly(2,3dichlorobenzyl methacrylate): circles and continuous line represent the experimental and calculated results using the Williams-Watts ansatz [Eq. (24)], respectively. The dash curves correspond to the contributions of the $\alpha$ (low frequency) and $\beta$ (high frequency) relaxations.

$T_{\mathrm{co}}$, the partial relaxation in the local environments must surmount barrier energies nearly two times larger than those processes occurring in the local environments existing at $T>T_{\text {co. }}$. As a consequence, above the crossover temperature, the strength of $\beta$ experiences a sharp increase with increasing temperature. The fact that the activation energy associated with the $\alpha$ relaxation at $T>T_{\text {co }}$ is similar to that of the $\beta$ process suggests that both relaxations are not independent in this region, but they are strongly correlated leading to a significant decrease of the cooperativity level of the $\alpha$ relaxation in the $\alpha \beta$ zone. Segmental motions play a major role in the relaxation polarization below $T_{\mathrm{co}}$, but its contribution to the relaxation is lower than that of local motions at $T>T_{\mathrm{co}}$.

The distance between the deconvoluted $\alpha$ and $\beta$ peaks, expressed in terms of $\log \left(f_{\max ; \beta} / f_{\max ; \alpha}\right)$, where $f_{\max }$ is the frequency at each peak maximum, is plotted against the reciprocal of the absolute temperature in Fig. 11. It is worth noting that except for the isotherms corresponding to extreme low and high temperatures, the temperature dependence of the distance obeys fairly well to Arrhenius behavior, even though the $\alpha$ relaxation is not a thermally activated process but it is governed by the volume. At high temperature, the distance between the peaks remains constant presumably as a consequence of the fact that the $\alpha$ relaxation in this range of temperatures behaves like a thermally activated process with an activation energy similar to that of the $\beta$ absorption above the crossover temperature.

The stretch exponent $\beta_{\mathrm{KWW}}$ of the KWW equation describing the deconvoluted $\alpha$ relaxations in the time domain are shown in Fig. 14. The exponent at temperatures slightly higher than $T_{g}$ is small, suggesting a rather wide distribution of retardation times. However, $\beta_{\mathrm{KWw}}$ increases as temperature increases reaching a value of ca. 0.40 at $380 \mathrm{~K}$, similar to that found for the parameter accounting for the width of the distribution of the $\beta$ process at similar temperature.

The Williams ansatz holds in a wide range of temperature though contrary to what occurs in other systems having the $\alpha$ and $\beta$ processes highly overlapped, for which 
$\phi^{*}(\omega) \cong \phi_{\alpha}^{*}(\omega) \phi_{\beta}^{*}(\omega),{ }^{14}$ a relatively high fraction of the mean-square dipole moment still relaxes exclusively through the $\alpha$ process. As Fig. 14 shows, the value of $f_{\alpha}$ moderately decreases with increasing temperature. At high temperatures, where the temperature dependence of the $\alpha$ relaxation approaches Arrhenius behavior, the Williams ansatz no longer holds. This fact, together with the existence of a crossover temperature in the $\beta$ process and the absence of the onset observed in the $\alpha$ relaxation of poly( $n$-alkyl methacrylate)s (Refs. 18 and 19) and poly(5-acryloxy-5-methyl-1,3dioxacyclohexane) (Ref. 20) could be explained assuming the configurational space above $T_{g}$ as formed by craters and basins, the latter located not only in the space between the craters but also inside the craters. The $\alpha$ and $\beta$ relaxations would take place across the depth craters and the basins, respectively. ${ }^{42}$ As temperature decreases, the craters become rare until a temperature is reached at which the $\alpha$ relaxation cannot occur, remaining below $T_{g}$ only the basins through which the $\beta$ process relaxes. At high temperatures the depths of both the craters and the basins decrease but difference between them still persists avoiding the disappearance of the $\alpha$ relaxation in the range of temperatures investigated.

\section{CONCLUSIONS}

The analysis of the dielectric broadband experiments performed in terms of the retardation spectra allows a rather unbiased deconvolution of the overlapped $\alpha$ and $\beta$ relaxations. The temperature dependence of the secondary relaxation reveals the existence of a crossover reflected in a decrease of the activation energy of the local processes above the crossover temperature, which produces an enhancement of the $\beta$ absorption. The relaxation strength of the $\alpha$ relaxation slightly decreases with increasing temperature, but the onset temperature found for this process in most systems seems to be absent in this polymer. The distance between the $\alpha$ and $\beta$ relaxations expressed as $\log \left(f_{\max ; \beta} / f_{\max ; \alpha}\right)$ follows Arrhenius behavior below the crossover temperature of the $\beta$ process presumably as a consequence of the fact that the activation energies of the both processes become similar. The Williams ansatz only holds below the crossover temperature. This and other studies suggest that the relaxation behavior of the condensed matter at high temperature is strongly conditioned by the fine chemical structure.

\section{ACKNOWLEDGMENTS}

This work was financially suported by the DGCYT and CAM through Grant Nos. MAT2002-04042-C02 and GR/ MAT/0723/2004. Two of the authors (L.G. and D.R.) thank the FONDECYT for partial financial support through Grant Nos. 21050956 and 1050962, respectively.

\footnotetext{
${ }^{1}$ For a review, P. Lunkenheimer, U. Schneider, R. Brand, and A. Loidl, Contemp. Phys. 41, 15 (2000), and references therein.

${ }^{2}$ G. P. Johari and M. Goldstein, J. Chem. Phys. 53, 2372 (1979).

${ }^{3}$ G. P. Johari and M. Goldstein, J. Phys. Chem. 74, 2034 (1970); J. Chem. Phys. 55, 4245 (1971).

${ }^{4}$ N. G. McCrum, B. E. Read, and G. Williams, Anelastic and Dielectric Effects in Polymeric Solids (Wiley, London, 1967).
}

${ }^{5}$ G. Adam and J. H. Gibbs, J. Chem. Phys. 43, 139 (1965).

${ }^{6}$ M. H. Cohen and G. S. Grest, Phys. Rev. B 20, 1077 (1979).

${ }^{7}$ K. L. Ngai, Comments Solid State Phys. 9, 127 (1979); 9, 149 (1979); in Disorder Effects on Relaxational Processes, edited by R. Richert and A. Blumen (Springer, Berlin, 1994), p. 89, and references therein.

${ }^{8}$ U. Bengtzelius, W. Götze, and A. Sjolander, J. Phys. C 17, 5915 (1984); E. Leutheusser, Phys. Rev. A 29, 2765 (1984).

${ }^{9}$ D. Kivelson, S. A. Kivelson, X-L. Zhao, Z. Nussinov, and G. Tarjus, Physica A 219, 27 (1995); G. Tarjus, D. Kivelson, and S. A. Kiveslson, in Supercooled Liquids, Advance and Novel Applications, ACS Symposium Series, edited by J. T. Fourkas, D. Kivelson, U. Mohanty, and K. A. Nelson (American Chemical Society, Washington, DC, 1997), Vol. 676, p. 67.

${ }^{10}$ R. V. Chamberlin, Phys. Rev. Lett. 82, 2520 (1999).

${ }^{11}$ For a review, H. Sillescu, J. Non-Cryst. Solids 243, 81 (1999), and references therein.

${ }^{12}$ J. P. Garrahan and D. Chandler, Phys. Rev. Lett. 89, 035704 (2002); J. P. Garrahan and D. Chandler, Proc. Natl. Acad. Sci. U.S.A. 100, 9710 (2003); Y-J. Jung, J. P. Garrahan, and D. Chandler, Phys. Rev. E 69, 061205 (2004).

${ }^{13}$ Y. N. Huang, C. J. Wang, and E. Riande, J. Chem. Phys. 122, 144502 (2005).

${ }^{14}$ D. Gómez, A. Alegría, and J. Colmenero, Macromolecules 34, 503 (2001).

${ }^{15}$ G. Williams, Adv. Polym. Sci. 33, 60 (1979).

${ }^{16}$ G. Williams, in Comprehensive Polymer Science, edited by G. Allen and J. C. Bevington (Pergamom, Oxford, 1989), Vol. 2, Chap. 7, p. 601.

${ }^{17}$ G. Williams, in Keynote Lectures in Selected Topics of Polymer Science, edited by E. Riande (CSIC, Madrid, 1995), Chap. 1.

${ }^{18}$ F. Garwe, A. Schönhals, H. Lockwenz, M. Beiner, K. Schröter, and E. Donth, Macromolecules 29, 247 (1996).

${ }^{19}$ K. Schröter, R. Unger, S. Reissig, F. Garwe, S. Kahle, M. Beiner, and E. Donth, Macromolecules 31, 8966 (1998).

${ }^{20}$ Y.-N. Huang, E. Saiz, T. Ezquerra, J. Guzmán, and E. Riande, Macromolecules 35, 2926 (2002); C. Álvarez, V. Lorenzo, and E. Riande, J. Chem. Phys. 122, 194905 (2005).

${ }^{21}$ S. Havriliak, Jr. and S. J. Havriliak, Dielectric and Mechanical Relaxation in Polymers (Hanser, New York, 1997), Chap. 1.

${ }^{22}$ R. Kohlrausch, Ann. Phys. 12, 3931 (1847).

${ }^{23}$ G. Williams and D. D. Watts, Trans. Faraday Soc. 66, 80 (1970).

${ }^{24}$ F. Álvarez, A. Alegría, and J. Colmenero, Phys. Rev. B 44, 7306 (1991); 47, 125 (1993).

${ }^{25}$ R. Díaz-Calleja, Macromolecules 33, 8924 (2000).

${ }^{26}$ E. Riande and R. Díaz-Calleja, Electrical Poperties of Polymers (Marcel Dekker, New York, 2004), Chap. 8.

${ }^{27}$ W. H. Press, S. A. Teukolsky, W. T. Vetterling, and B. P. Flannerty, Numerical Recipes in C: The Art of Scientific Computing, 2nd ed. (Cambridge University Press, New York, 1992), Chap. 18.

${ }^{28}$ V. A. Morozov, Methods for Solving Incorrectly Posed Problems (Springer, New York, 1984).

${ }^{29}$ S. W. Provencher, Comput. Phys. Commun. 27, 229 (1982).

${ }^{30}$ A. Reiner Zorn, J. Polym. Sci., Part B: Polym. Phys. 37, 1043 (1999).

${ }^{31}$ R. Fuoss and J. G. Kirkwood, J. Am. Chem. Soc. 63, 385 (1941).

${ }^{32}$ J. Ross Macdonald, Impedance Spectroscopy (Wiley-Interscience, New York, 1987), p. 41.

${ }^{33}$ H. Z. Vogel, Z. Phys. 22, 645 (1921); J. S. Fulcher, J. Am. Ceram. Soc. 8, 839 (1925); G. Tammann and W. Z. Hesse, Z. Anorg. Allg. Chem. 156, 245 (1926).

${ }^{34}$ C. A. Angell, Science 267, 1935 (1995).

${ }^{35}$ E. Donth, Relaxations and Themodynamics in Polymers: Glass Transition (Akademic, Berlin, 1992).

${ }^{36}$ A. K. Doolittle and D. B. Doolittle, J. Appl. Phys. 28, 901 (1957).

${ }^{37}$ M. H. Cohen and D. J. Turnbull, Chem. Phys. 30, 748 (1959).

${ }^{38}$ J. D. Ferry, Viscoelastic Properties of Polymers, 2nd ed. (Interscience, New York, 1972), p. 316.

${ }^{39}$ E. Arbe, D. Richter, J. Colmenero, and B. Farago, Phys. Rev. E 54, 3853 (1996).

${ }^{40}$ E. Riande and E. Saiz, Dipole Moments and Birefringence of Polymers (Prentice Hall, Englewood Cliffs, NJ, 1992).

${ }^{41}$ R. Bergman, F. Alvarez, A. Alegría, and J. Colmenero, J. Chem. Phys. 109, 7546 (1998).

${ }^{42}$ F. F. Stillinger, Science 267, 1924 (1995). 